\title{
Transcription Table: Text Support During Meetings
}

\author{
Joris van Gelder, Irene van Peer, and Dzmitry Aliakseyeu
}

\author{
Faculty of Industrial Design, \\ Eindhoven University of Technology, \\ Den Dolech 2, Eindhoven, The Netherlands \\ J.M.v.Gelder@student.tue.nl, \{i.p.v.peer, d.aliakseyeu\}@tue.nl
}

\begin{abstract}
In this paper we present the design of a tool that allows a hearingimpaired person to participate in a meeting. The tool combines speech recognition with a table top augmented reality system. One of the foremost requirements was that the tool should not stigmatize the hearing-impaired user. Therefore the tool supports features that are attractive to all participants in the meeting. The final prototype has the following features: it can convey (some of) the meeting content to the hearing-impaired person, it supports eye contact between participants, it allows an interaction with words and sentences, it provides a meeting history and instant minutes.
\end{abstract}

\section{Introduction}

Sudden deafness is a condition where someone who already knows how to talk becomes deaf at a later age [1]. Since the life of these people is not organized to function with deafness they usually are not able to continue their social and professional life and consequently suffer from isolation.

There are several communication products available for hearing-impaired people. Unfortunately none of them is a substitute for direct communication. One instance of professional activity where direct communication is very important is in meetings. When an employee can not participate in a meeting he/she has no share in many important decisions. An interview with several hearing-impaired people confirmed that this is indeed one of the biggest problems that they encounter.

We believe that by employing a combination of speech and tabletop augmented reality technologies we can at least partially resolve this problem. We particularly focus on small (work) meetings, since they are the most common meetings in a professional environment. Small meetings are the base for personal contact between co-workers and deal with the day-to-day organization of the work.

When developing a tool for such a group of users, an important requirement is that the tool should not stigmatize the hearing-impaired user. Therefore this project focuses not only on the perspective of the hearing-impaired user, but also on that of the other participants in the meeting. The tool should enable the hearing-impaired person to take part in a meeting and also provide an added value for the hearing participants of the meeting.

When all participants use the tool, the hearing-impaired person is not in a different position from the rest of the team. 
In summary, makes the design goals for the project are twofold: the tool has to be a translation device, i.e. convey (some of) the meeting content to the hearing-impaired, person and the tool needs to support the position of the hearing-impaired person in the group with features that are also attractive to the other participants in the meeting.

The remainder of the paper is organized as follows. First we describe a first exploratory user study that served to motivate some of the design choices. Next we discus the final design, expected problems and potential benefits.

\section{Exploratory User Study}

In order to study how people react on real-time transcriptions during a meeting, a wizard of $\mathrm{Oz}$ user study was performed. More precisely, we wanted to obtain answers to the following questions. First can a hearing-impaired person participate in a meeting using a speech-to-text translating device? Second, which influence does the added visual information have on the meeting?

The test consisted of two 15-minute sessions, with and without the transcription tool. Both sessions were video-taped and all participants were interviewed after the experiment. During the second session (without the transcriptions) the hearing participants used pen and paper to communicate their conversation to the hearingimpaired participant.

A simple tabletop projection setup was used. The transcriptions were displayed on the middle of the table in two orientations (toward and away from the speaker) so that participants on both sides of the table could read the transcriptions. The transcription subsequently moved towards the speaker.

Speech recognition was not implemented in this setup. Instead a human operator inserted transcriptions that were synchronized with the meeting.

The result of the exploratory study showed that: a hearing-impaired person was able to participate in the discussion; participants had little eye contact during the meeting; participants made little use of the transcriptions during their own story, they only read it when it appeared on the table. These results indicates that a possible tool, in addition to supporting transcription, should also stimulate and enable interaction with the words, and stimulate eye contact between the participants of the meeting.

\section{Prototype Design}

The results of the user study were used as a starting point for the prototype design.

From the user study, we conclude that interaction with the transcriptions is vital. To improve this interaction, we decided that text feedback should be displayed within hand reach of the speaker. Such text position can also stimulate eye contact (see Figure 1).

In addition to the eyes, the hands are an important communication tool. With their hands, users can emphasize words and sentences.

The transcription table has four functions: (I) Display the transcriptions for the users. (II) Display who is speaking. (III) Enable the participants to have interaction with the transcriptions. (IV) Hold items like cups of coffee or papers. 
The layout of the tabletop consists of a communication field (Figure 1.1), the soumaine, where the speaker can put his personal items (Figure 1.2), the touch screen (Figure 1.3) is the area where a speaker can see his own words and emphasize them by touch. This setup is equal for every participant of the meeting (Figure 1.4). Note that the current translation is only shown in the communication area of the speaker (Figure 1.5). Otherwise the other participants would not be able to see who is speaking. The transcription is displayed in multiple orientations to give all participants a comfortable view (Figure 1.6). The transcription is also displayed in the touch area of the speaker (Figure 1.7). When a transcription is replaced with a new transcription the old one is copied to the history which is also within the touch area (Figure 1.8). All participants have a copy of this history which consists of the four most recent sentences.

Two main points for interaction came out of the user study - the ability to emphasize words (sentences) and the ability to correct errors that can occur in the speech recognition. The users can emphasize sentences during the meeting. There are two types of emphasis, intonation and main clause emphasis. Intonation emphasis is the emphasis of single words to stress their importance or clarify their meaning. Intonation emphasis can only be executed by the speaker by touching the relevant word within the touch area. Main-clause emphasis is emphasizing a complete sentence to stress its importance. Main-clause emphasis can be executed by all participants of the meeting by touching a sentence in the history. The intonation emphasis still needs to be implemented in the prototype.
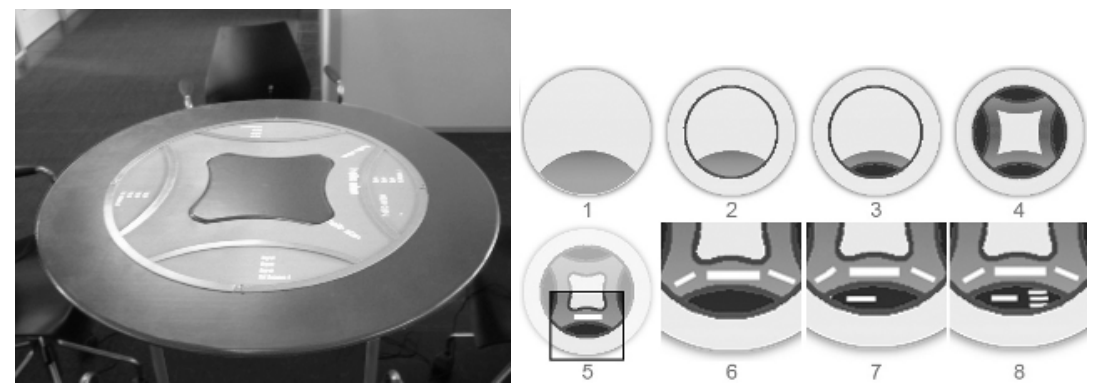

Fig. 1. Final prototype and step by step layout of the tabletop

Currently the speech recognition is controlled by a java program. The speech input is regulated by a microphone switch. The switch converts four microphone inputs into a single audio output. The input with the highest loudness is connected to the output.

The tool also provides instant minutes after the meeting. Since the computer has to translate all the spoken words into written sentences to be displayed on the table, they can also be saved. This transcription file can be printed right after the meeting is finished and be used as a basis for the minutes.

\section{Related Work}

There exists a lot of literature on supporting collaborative work with tabletop displays [7]. However most of this work does not consider speech as one of the input modali- 
ties. In terms of the technology, the most commonly used input devices are a touch sensitive surface (for example DiamondTouch [4]), or vision-based recognition [5].

Another area of related research is speech recognition, including the research on using speech recognition for disabled people [3]. Nevertheless there are not a lot of systems that combines both speech and tabletop display or touch input. One example is the Quickset [2], a speech/gesture multimodal system, which consists of parallel recognizers for the speech and gesture input modalities.

\section{Conclusions and Future Work}

The first prototype of the transcription table can convey (some of) the meeting content to the hearing-impaired person without stigmatizing him or her, it supports the eyecontact between participants, it allows an interaction with words and sentences, provides a meeting history and instant minutes.

A next step in this project is user testing with hearing-impaired users. Experiments with different turn taking setups would be interesting as well; an example is that the speaker remains connected to the computer as long as he is speaking.

\section{References}

1. Association of Late-Deafness Adults. http://www.alda.org/.

2. Cohen, P.R., Johnston, M., McGee, D.R., Oviatt, S.L., Pittman, J.A., Smith, I., et al. QuickSet: Multimodal interaction for distributed applications. In Proc. of the IEEE International Multimedia Conference, Seattle, WA, Nov. 1997), pp. 31-40.

3. Cox, s., Lincoln, M., Tryggvason, J., Nakisa, M., Wells, M., Tutt, M., Abbott, S. Tessa, a system to aid communication with deaf people. In Proc. of Assets'02, Edinburgh, Scotland, ACM Press (2002), pp. 205-212.

4. Deitz P., Leigh D. DiamondTouch: A Multi-User Touch Technology. In Proc. of UIST'01, Orlando, Florida, 2001, pp. 219-226.

5. Fitzmaurice, G. W., Ishii, H., Buxton, W. Bricks: Laying the Foundations for Graspable User Interfaces. In Proc. of CHI'95, May 1995, Denver, ACM Press (1995), pp. 442 - 449.

6. ScanSoft - Dragon NaturallySpeaking. http://www.scansoft.com/naturallyspeaking/

7. Scott, S.D., Grant, K.D., \& Mandryk, R.L. System Guidelines for Co-located, Collaborative Work on a Tabletop Display. In Proc. of ECSCW'03, European Conference ComputerSupported Cooperative Work 2003, Helsinki, Finland, 2003. 